\title{
Effective field theory of bosons with finite-range interaction in a disordered environment
}

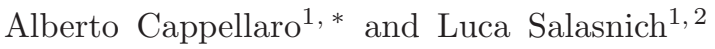 \\ ${ }^{1}$ Dipartimento di Fisica e Astronomia "Galileo Galilei" and CNISM, \\ Università di Padova, via Marzolo 8, 35131 Padova, Italy \\ ${ }^{2}$ CNR-INO, via Nello Carrara, 1 - 50019 Sesto Fiorentino, Italy
}

(Dated: May 1, 2020)

\begin{abstract}
We investigate the low-temperature properties of a ultracold gas made of bosonic alkali-metal atoms with finite-range interaction under the effect of a disordered environment. The statistical characterization of the disorder is investigated within an effective-field-theory formalism for a generic spatial dimension $d$. Moving to $d=3$, where all the arising divergences are properly regularized, we focus on the depletion of both the condensate and superfluid densities. At zero temperature we obtain meaningful analytical formulas for the condensate fraction and the superfluid fraction which take into account the interplay among scattering length, effective range, and disorder strength.
\end{abstract}

Keywords: cold atoms, superfluidity, disorder

\section{INTRODUCTION}

During the last decades, a great interest has been aroused by the interplay between interactions and disorder. The investigation of quantum effects in manyparticle systems moving in disordered environment goes back in time up to the prediction of a localized phase for free electrons in random-lattice structures [1].

Similarly to other condensed matter issues, significant efforts have been devoted to explore the role of a disordered environment in the framework of ultracold atomic gases [2, 3]. The main reason lies in the remarkable experimental control over the relevant physical parameters such as densities and interaction strengths. In order to construct a random potential mimicking a porous medium, a viable strategy consists in superimposing two different optical lattices [4, 5]. Experimentally, another possibility is provided by laser-speckle fields, arising from the interference pattern of waves with the same frequency but different phases and amplitudes [6 6 ] $]$.

In this paper we focus on disordered bosons within their superfluid phase but in presence of a finite-range interaction between the atoms. By taking into account a non-local two-body potential, nonuniversal corrections to the thermodynamic potential can be obtained beyond the usual mean-field picture [9 11]. As a consequence, detachments from universality may represent a viable approach to reveal the elusive contribution of quantum fluctuations. These corrections can also be included in a modified Gross-Pitaevskii equation (GPE), leading to nonuniversal dynamical features 12 16].

Our analysis is carried on within the framework of the effective-field-theory, where one can relate the coupling constants of the Euclidean action functional to the measurable s-wave scattering parameters, via the T-matrix technique [10, 17]. From a technical point of view, in

*Electronic address: cappellaro@pd.infn.it order to implement a quantum field theory in presence of a disorder (external) potential, different strategies are at disposal. Since we are interested in how disorder and finite-range interactions both contribute to modify the condensate and superfluid depletion, we adopt a perturbative approach: the disorder is assumed to be weak, in a similar way as quantum and thermal fluctuations. Their role is then taken into account up to the one-loop (i. e. Gaussian) level. For a zero-range interaction, it has been shown that also a very weak disorder affects the thermodynamic picture of a bosonic superfluid [ 18 20]

On the other hand, the case of strong disorder has to be treated non-perturbatively. The most standard approaches all rely upon the so-called replica trick, first proposed in the context of spin glasses [21 24]. In [25], a replicated Hartree-Fock theory managed to reproduce the phase diagram for interacting bosons where also the localized phase is included. However, in this paper we are going to focus only on the weak disorder regime, where the replica trick is not crucial, since it basically reproduces the perturbative results, as shown for dirty superconductors [26] and cold atoms [27].

The paper is organized as follows: first we review how (weak) disorder can be included within an Euclidean functional formulation for a system of interacting bosons in a generic dimension $d$. Then we move to compute the thermodynamic potential up to the Gaussian level in quantum and thermal fluctuations, by taking into account both the presence of an uncorrelated quenched disorder and a finite-range interaction between the atoms. In order to simplify the computational burden, we focus on point-like defects. The case $d=3$ is analyzed in more detail and explicit results are given also for the thermal depletion of the superfluid density.

\section{MODELLING THE DISORDER}

In this paper, we study the interplay between disorder, non-local interaction and fluctuations in a quantum gas 
made of bosonic particles. To fulfill this task, our analysis will be carried on within the functional integration framework.

In order to properly construct the path integral for an ensemble of identical atomic bosons with mass $m$, we start from the second-quantized hamiltonian

$$
\begin{aligned}
\hat{K} & =\int_{L^{d}} d^{d} \mathbf{r} \hat{\psi}^{\dagger}(\mathbf{r})\left[-\frac{\hbar^{2} \nabla^{2}}{2 m}-\mu-U_{D}(\mathbf{r})\right] \hat{\psi}(\mathbf{r}) \\
& +\frac{1}{2} \int_{L^{d}} d^{d} \mathbf{r} d^{d} \mathbf{r}^{\prime} \hat{\psi}^{\dagger}(\mathbf{r}) \hat{\psi}^{\dagger}\left(\mathbf{r}^{\prime}\right) V\left(\left|\mathbf{r}-\mathbf{r}^{\prime}\right|\right) \hat{\psi}\left(\mathbf{r}^{\prime}\right) \hat{\psi}(\mathbf{r})
\end{aligned}
$$

where $V(r)$ is the central two-atom interaction potential (with $r=|\mathbf{r}|$ ), while $U_{D}(\mathbf{r})$ is the random external potential, which takes into account the effect of disordered environment. The field operators in Eq. (11) respect the usual bosonic commutation relation $\left[\hat{\psi}(\mathbf{r}), \hat{\psi}^{\dagger}\left(\mathbf{r}^{\prime}\right)\right]=$ $\delta^{(d)}\left(\mathbf{r}-\mathbf{r}^{\prime}\right)$. The spatial integration is taken over a large $d$-dimensional ipercubic volume $L^{d}$; at the end of calculation, if interested in the thermodynamic limit, we have to take the limit $L \rightarrow \infty$.

Given Eq. (11) and adopting the functional intergation formalism, we can represent the partition function of the system as 28]

$$
\mathcal{Z}=e^{-\beta \Omega}=\int \mathcal{D}\left[\psi, \psi^{*}\right] \exp \left\{-\frac{1}{\hbar} S_{E}\left[\psi, \psi^{*}\right]\right\}
$$

where $\Omega$ is the thermodynamic potential, $\psi(\mathbf{r}, \tau)$ is the coarse-grained complex field modelling the bosons, $\mathcal{D}\left[\psi, \psi^{*}\right]$ is the measure of the functional integration over $\psi(\mathbf{r}, \tau)$ and $\psi^{*}(\mathbf{r}, \tau)$, and $S_{E}\left[\psi, \psi^{*}\right]$ denotes the Euclidean (i.e. imaginary time) action defined as

$$
S_{E}\left[\psi, \psi^{*}\right]=\int_{0}^{\beta \hbar} d \tau \int_{L^{d}} d^{d} \mathbf{r}\left[\psi^{*} \hbar \partial_{\tau} \psi+K\left(\psi, \psi^{*}\right)\right] .
$$

In the equation above $\beta=1 /\left(k_{B} T\right), k_{B}$ being the Boltzmann constant and $T$ the absolute temperature.

It is worth to characterize the external random potential $U_{D}(\mathbf{r})$ from a statistical point of view. This implies that its features can be extracted from a probability density function (PDF) according to which single realizations of a disordered environment are distributed. A reasonable choice for $U_{D}(\mathbf{r})$ can be a centered (i.e. zero average) Gaussian as the following one

$$
\mathcal{P}\left[U_{D}\right] \propto \exp \left\{-\frac{1}{2} \int d^{d} \mathbf{r} d^{d} \mathbf{r}^{\prime} U_{D}(\mathbf{r}) \Delta^{-1}\left(\mathbf{r}, \mathbf{r}^{\prime}\right) U_{D}\left(\mathbf{r}^{\prime}\right)\right\}
$$

with a proper normalization factor which is, however, not important in the proceeding [28]. Indeed, we will mostly need the correlator

$$
\left\langle U_{D}(\mathbf{r}) U_{D}\left(\mathbf{r}^{\prime}\right)\right\rangle_{\text {dis }}=\Delta\left(\mathbf{r}, \mathbf{r}^{\prime}\right)
$$

where $\langle\ldots\rangle_{\text {dis }}$ has to be intended as

$$
\langle\ldots\rangle_{\mathrm{dis}}=\int \mathcal{D}\left[U_{D}\right] \mathcal{P}\left[U_{D}\right](\ldots) .
$$

From Eq. (44) it is clear that we are assuming that the characteristic time scale of $U_{D}(\mathbf{r})$ is infinitely long compared to the other ones; we are then restricting ourselves to the (important) case of a quenched disordered environment. Obviously, this assumption can be relaxed and the random potential can be taken as time- or temperaturedependent [29].

Among Gaussian-distributed random configurations, the most simple situation is provided by potentials with null correlation length. They all have a $\delta$-like correlator, such

$$
\Delta\left(\mathbf{r}, \mathbf{r}^{\prime}\right)=\Delta(\mathbf{r}) \delta^{(d)}\left(\mathbf{r}-\mathbf{r}^{\prime}\right) .
$$

Despite being an extremely simplifying assumption, the equation above suits well with disorder generated by static and point-like bosonic impurities [29]. More complicated disordered configurations are discussed, for instance, in [29, 30].

\section{DISORDER AND FINITE-RANGE INTERACTIONS}

\section{A. The finite-range effective potential}

The crucial issue now consists in a proper choice of the two-body interaction. Since the first seminal experiments with the alkali atoms in the degenerate regime, it appeared that reliable analysis and predictions can be carried on by considering a contact interactions (i.e. zero-range) $V(r)=g_{0} \delta^{(3)}(r)$, where $r=|\mathbf{r}|$ and $g_{0}=$ $\int d^{d} \mathbf{r} V(r)$ is the interaction strength. This approximation leads to a universal thermodynamics, where all the relevant equilibrium features of the system depend only on the coupling parameter [31, 32].

However, thanks to Feshbach resonances, it is possible to explore regimes where deviations from universalities become relevant [10 12, 15, 33]. Moreover, in [9] it was already pointed out that some divergences in the perturbative expansion of the thermodynamic potential of Bose gases can be healed by involving additional features of the interaction potential such as its characteristic range.

In order to analyze the first correction to the thermodynamic properties of the Bose gas due to a finite-range interaction, we begin by considering the following lowmomentum expansion

$$
\tilde{V}(q)=g_{0}+g_{2} q^{2}+\mathcal{O}\left(q^{4}\right),
$$

of the Fourier transform $\tilde{V}(q)$ of two-body interaction potential $V(r)$, where $g_{0}=\tilde{V}(0)$ and $g_{0}=(1 / 2) \tilde{V}^{\prime \prime}(0)$.

By making use of the effective field theory techniques [9] and of the T-matrix formulation of two-atoms collisions [17], it is possible to define the coupling constants $g_{0}$ and $g_{2}$ in the equation above in terms of measurable scattering parameters. As discussed in detail in Ref. [11], one obtains

$$
g_{0}=\frac{4 \pi \hbar^{2}}{m} a_{s}, \quad g_{2}=\frac{2 \pi \hbar^{2}}{m} a_{s}^{2} r_{s} .
$$


It is worth recalling that this recipe can be likewise applied in lower dimensions, namely $d=1$ and (with some technical complications) in $d=2[33]$.

As mentioned above, thanks to Feshbach resonances, one can explore a wide range of values for the $s$-wave scattering length by tuning an external magnetic fields. The effective range expansion responsible for Eqs. (8) and (9) has to be treated carefully close to narrow resonances or zero crossings of $a_{s}$ as a function of the magnetic field $B$. Rigorously speaking, close to a narrow resonance, a multichannel effective theory should be required, as detailed in [34], naturally reading to finite-range effects. Other multi-channel approaches rely upon the formation of formation of molecular bound states in the regime of resonant interaction 35 37.

However, it has been shown that it is possible to reduce the multi-channel scattering problem to a single-channel one [38], at least for a broad resonance. The analysis reported in [38] is further extended for zero-point crossings and narrow resonances in [39] where it is also compared with multichannel numerical outcomes. Remarkably, for the isolated resonances in ${ }^{39} \mathrm{~K}$ and ${ }^{133} \mathrm{Cs}$ a very good agreement is found, providing a very good single-channel approximation.

\section{B. $\delta$-correlated Gaussian disorder: perturbative analysis}

At the mean-field level, the space-time translationallyinvariant ground state of the system can be described by a constant $v$, namely $\psi(\mathbf{r}, \tau)=v$. For the sake of simplicity the constant $v$ is taken real. By inserting it in Eq. (3), the mean-field thermodynamic potential reads

$$
\frac{\Omega_{\mathrm{mf}}(\mu, v)}{L^{d}}=-\left[\mu+\left\langle U_{D}\right\rangle_{\mathrm{dis}}\right] v^{2}+\frac{1}{2} \tilde{V}(0) v^{4},
$$

where we have already considered the disorder average defined in Eq. (6). However, in the following, we are going to consider the case of $\delta$-correlated centered Gaussian disorder, so $\left\langle U_{D}\right\rangle_{\text {dis }}=0$. Eq. (10) is stationarized only by $v=0$ for $\mu<0$, but $v$ acquires a non-zero value for $\mu>0$ :

$$
v^{2}=\frac{\mu}{\tilde{V}(0)}
$$

This fact obviously signals the occurring of the superfluid transition, where a $U(1)$ symmetry is spontaneously broken.

It is important to remark that a $\delta$-correlated does not affect the mean-field picture of the homogeneous and stationary ground state. Moreover, if we consider the interaction potential as given by Eq. (8), we immediately realize that only $g_{0}=\tilde{V}(0)$ appears up to this level of approximation. As a consequence, in order to explore the role of disorder and nonlocal interactions, one has to consider fluctuations, both quantum and thermal, above the ground state given by Eq. (11).

Let us then consider the following shift of the field

$$
\psi(\mathbf{r}, \tau)=v+\eta(\mathbf{r}, \tau)
$$

with $\eta(\mathbf{r}, \tau)$ being the complex fluctuating field.

By replacing Eq. (12) in the Euclidean action $S_{E}\left[\psi, \psi^{*}\right]$ and retaining terms up to the quadratic (Gaussian) level in $\eta$ and $\eta^{*}$, the partition function defined in Eq. (2) can be factorized in

$$
\mathcal{Z}\left[U_{D}\right] \simeq e^{-\beta \Omega_{\mathrm{mf}}} \int \mathcal{D}\left[\eta, \eta^{*}\right] e^{-\frac{1}{\hbar}\left(S_{g}^{(\text {pure })}[v, \eta]+S_{g}^{(\mathrm{dis})}\left[v, \eta, U_{D}\right]\right)}
$$

where $\Omega_{\mathrm{mf}}$ is given by Eq. (10), $S_{g}^{(\text {pure) }}$ is the Euclidean action describing the periodic imaginary-time trajectories of the fluctuating fields $\eta$ and $\eta^{*}$, while the disorder contribution is encoded in $S_{g}^{\text {(dis) }}$.

Within our perturbative scheme, $S_{g}^{(\text {pure) }}$ reads the same expression for a system not subject to an external disordered environment. In the Fourier space, this implies that

$$
S_{g}^{(\text {pure })}=\frac{\hbar}{2} \sum_{\mathbf{q}, \omega_{n}} \Psi^{\dagger}\left(\mathbf{q}, \omega_{n}\right) \mathbb{M}\left(\mathbf{q}, \omega_{n}, v\right) \Psi\left(\mathbf{q}, \omega_{n}\right)
$$

with the spinor $\Psi^{\dagger}\left(\mathbf{q}, \omega_{n}\right)=\left[\tilde{\eta}^{*}\left(\mathbf{q}, \omega_{n}\right), \tilde{\eta}\left(-\mathbf{q},-\omega_{n}\right)\right]$ and

$$
\mathbb{M}\left(\mathbf{q}, \omega_{n}, v\right)=\beta\left(\begin{array}{cc}
-i \hbar \omega_{n}+\frac{\hbar^{2} q^{2}}{2 m}-\mu+g_{0} v^{2}+v^{2}\left(g_{0}+g_{2} q^{2}\right) & v^{2}\left(g_{0}+g_{2} q^{2}\right) \\
v^{2}\left(g_{0}+g_{2} q^{2}\right) & i \hbar \omega_{n}+\frac{\hbar^{2} q^{2}}{2 m}-\mu+g_{0} v^{2}+v^{2}\left(g_{0}+g_{2} q^{2}\right)
\end{array}\right)
$$


Matsubara ones, defined as $\omega_{n}=2 \pi n /(\beta \hbar)$ [28].

On the other hand, the disorder contribution $S_{g}^{(\text {dis })}$ can be written down as [40]

$$
S_{g}^{(\mathrm{dis})}=-v \int_{0}^{\beta \hbar} d \tau \int d^{d} \mathbf{r} U_{D}(\mathbf{r})(1,1) \cdot\left(\begin{array}{c}
\eta(\mathbf{r}, t) \\
\eta^{*}(\mathbf{r}, t)
\end{array}\right) .
$$

Since Eq. (13) has, by construction, a Gaussian structure, the functional integration over $\eta$ and $\eta^{*}$ can be performed exactly, reading, in Fourier space [27],

$$
\begin{aligned}
\mathcal{Z}\left[U_{D}\right] & \simeq e^{-\beta \Omega_{\mathrm{mf}}} \exp \left\{-\frac{1}{2} \sum_{\mathbf{q}, \omega_{n}} \log \left[\operatorname{det} \mathbb{M}\left(q, \omega_{n}\right)\right]\right\} \\
& \times \exp \left\{\frac{1}{2} \sum_{\mathbf{q}}\left|\tilde{U}_{D}(\mathbf{q})\right|^{2}(v, v) \mathbb{M}^{-1}(\mathbf{q}, 0)\left(\begin{array}{l}
v \\
v
\end{array}\right)\right\},
\end{aligned}
$$

where $\tilde{U}_{D}(\mathbf{q})$ is the Fourier transform of the random potential $U_{D}(\mathbf{r})$. Let us remark that, in the equation above, the propagator $\mathbb{M}$ is computed at $\omega_{n}=0$, so there is no dependency on the temperature in the disorder contribution. As stated in Sec. II this is due to the assumption of a quenched disorder, whose characteristic features are frozen compared to other (quantum and thermally) fluctuating quantities.

The pure contribution of Gaussian fluctuations leads us to

$$
\Omega_{g}^{(\text {pure })}(\mu, v)=\frac{1}{2 \beta} \sum_{\mathbf{q}, \omega_{n}} \log \left[\beta^{2}\left(\hbar^{2} \omega_{n}^{2}+E_{q}^{2}\right)\right]
$$

with

$$
E_{q}^{2}=\left[\frac{\hbar^{2} q^{2}}{2 m}-\mu+g_{0} v^{2}+v^{2} \tilde{V}(q)\right]^{2}-v^{4} \tilde{V}^{2}(q)
$$

being the elementary excitation spectrum over the uniform ground state. By performing the Matsubara summation in Eq. (18) we can identify the quantum and thermal contribution to $\Omega_{g}^{\text {(pure) }}$ :

$$
\Omega_{g}^{\text {(pure) }}=\frac{1}{2} \sum_{\mathbf{q}} E_{q}+\frac{1}{\beta} \sum_{\mathbf{q}} \log \left(1-e^{-\beta E_{q}}\right) .
$$

Clearly, $\Omega_{g}^{\text {(pure) }}$ is independent from $U_{D}(\mathbf{r})$, so Eq. (20) is left untouched by the (functional) disorder average introduced in Eq. (6), i.e. $\left\langle\Omega_{g}^{\text {(pure) }}\right\rangle_{\text {dis }}=\Omega_{g}^{\text {(pure) }}$. Obviously, this is not true for disorder term. Indeed, if we take the logarithm of the second line of Eq. (13) we get the corresponding

$$
\Omega_{g}^{(\mathrm{dis})}=-\frac{v^{2}}{2} \sum_{\mathbf{q}}\left|\tilde{U}_{D}(\mathbf{q})\right|^{2}(1,1) \cdot \mathbb{M}^{-1}(\mathbf{q}, 0)\left(\begin{array}{l}
1 \\
1
\end{array}\right)
$$

Under the assumption of a Gaussian distribution disorder, $\left\langle\Omega_{g}^{(\text {dis })}\right\rangle_{\text {dis }}$ can be easily computed by using Eq. (5), which gives

$$
\int \mathcal{D}\left[U_{D}\right] \mathcal{P}\left[U_{D}\right]\left|U_{D}(\mathbf{q})\right|^{2}=\left\langle\left|\tilde{U}_{D}(\mathbf{q})\right|^{2}\right\rangle_{\mathrm{dis}}=\tilde{\Delta}(\mathbf{q}),
$$

where $\tilde{\Delta}(\mathbf{q})$ is the Fourier transform of the deltacorrelated disorder field $\Delta(\mathbf{r})$. In this way we obtain

$$
\left\langle\Omega_{g}^{(\mathrm{dis})}\right\rangle_{\mathrm{dis}}=-\frac{v^{2}}{2} \sum_{\mathbf{q}} \tilde{\Delta}(\mathbf{q})(1,1) \cdot \mathbb{M}^{-1}(\mathbf{q}, 0)\left(\begin{array}{l}
1 \\
1
\end{array}\right)
$$

The inverse of Eq. (15) is immediate to compute, such that, at the continuum limit $\sum_{\mathbf{q}} \rightarrow L^{d} \int d^{d} \mathbf{q} /(2 \pi)^{d}$,

$$
\frac{\left\langle\Omega_{g}^{(\mathrm{dis})}\right\rangle_{\mathrm{dis}}}{L^{d}}=-v^{2} \int \frac{d^{d} \mathbf{q}}{(2 \pi)^{d}} \frac{\tilde{\Delta}(\mathbf{q})}{\frac{\hbar^{2} q^{2}}{2 m}+2 v^{2} g_{2} q^{2}-\mu+3 g_{0} v^{2}} .
$$

In the following section we derive analytical and numerical results for the disorder contribution to the thermodynamic potential provided Eq. (24) as a starting point.

\section{POINT-LIKE DEFECTS}

\section{A. Disorder contributions in d dimensions}

In order to proceed with our calculation, it is necessary to specify the spatial behaviour (or, equivalently, the dependence on $\mathbf{q}$ ) of the disorder correlator $\Delta(\mathbf{r})$. For point-like defects, Eq. (7) is further simplified assuming

$$
\tilde{\Delta}(\mathbf{q})=\Delta,
$$

where $\Delta$ is a real constant. As mentioned earlier, despite this simple assumption, the system, in its superfluid phase is nevertheless crucially affected by the presence of a disordered external environment.

In order to highlight this feature, given Eq. (25), let us recast Eq. (24) as

$$
\frac{\left\langle\Omega_{g}^{(\mathrm{dis})}\right\rangle_{\mathrm{dis}}}{L^{d}}=-\frac{\Delta v^{2}}{\lambda\left(v^{2}, g_{2}\right)} \int \frac{d^{d} \mathbf{q}}{(2 \pi)^{d}} \frac{1}{\frac{\hbar^{2} q^{2}}{2 m}+\frac{3 g_{0} v^{2}-\mu}{\lambda\left(v^{2}, g_{2}\right)}}
$$

where, in analogy with the notation used in [11], we have defined

$$
\lambda\left(v^{2}, g_{2}\right)=1+\frac{4 m g_{2}}{\hbar^{2}} v^{2}
$$

At this point, it is immediate to realize that the disorder contribution $\left\langle\Omega_{g}^{(\text {dis })}\right\rangle_{\text {dis }} / L^{d}$ diverges for $d \geq 2$. However, it has been extensively shown (see [10, 32, 41] for technical details) that (finite) meaningful information may be extracted by means of the so-called dimensional regularization.

The key point of this method consists in performing the integration in Eq. (26) in a generic complex dimension $d_{\epsilon}=d-\epsilon$ with values of $\epsilon \in \mathbb{C}$ for which the result is convergent. The last step requires an analytical continuation back to the physical dimension we are interested in, i.e. one to consider a proper limit procedure such 
that $\epsilon \rightarrow 0$. Within this regularization framework, the following result holds

$$
\mathcal{I}(d)=\int \frac{d^{d} \mathbf{q}}{(2 \pi)^{d}} \frac{1}{q^{2}+M^{2}}=\frac{\Gamma(1-d / 2)}{2^{d} \pi^{d / 2}} M^{d-2},
$$

$\Gamma(z)$ being the Euler's Gamma function. As a consequence, Eq. (26) reads

$$
\begin{array}{r}
\frac{\left\langle\Omega_{g}^{(\mathrm{dis})}\right\rangle_{\mathrm{dis}}}{L^{d}}=-\frac{\Gamma(1-d / 2)}{(2 \pi)^{d / 2}\left(\frac{m}{\hbar^{2}}\right)^{d / 2}} \\
\frac{\Delta v^{2}\left(3 g_{0} v^{2}-\mu\right)^{d / 2-1}}{\lambda^{d / 2}\left(v^{2}, g_{2}\right)} .
\end{array}
$$

Moving from Eq. (29), a simple derivative leads us to the disorder contribution to the total density as a function of the condensed one. More technically,

$$
\begin{aligned}
n_{g}^{(\mathrm{dis})}\left(n_{0}\right) & =-\left.\frac{1}{L^{d}} \frac{\partial}{\partial \mu}\left\langle\Omega_{\text {dis }}^{(0)}(v, \mu)\right\rangle_{\operatorname{dis}}\right|_{\substack{\mu=g_{0} n_{0} \\
v^{2}=n_{0}}} \\
& =\frac{\Gamma(2-d / 2)}{4 \pi^{d / 2}}\left(\frac{m}{\hbar^{2}}\right)^{d / 2} \frac{\Delta g_{0}^{d / 2-2} n_{0}^{d / 2-1}}{\lambda^{d / 2}\left(n_{0}, g_{2}\right)} .
\end{aligned}
$$

It is fundamental to underline that, following a perturbative scheme, we have identified $v^{2}$ as given in Eq. (11) with the density $n_{0}$ of condensed atoms. Thus, our approach provides an implicit expression for the condensate density of the system. Since the pure and the disorder contributions to the thermodynamic potential are additive, as evident from Eqs. (13) and (20), the same occurs for the contributions to the total number density, i.e.

$$
n=n_{0}+n_{g}^{(0)}(n)+n_{g}^{(T)}(n)+n_{g}^{(\mathrm{dis})}(n) .
$$

Let us also point out that, within a perturbative approach for the weakly interacting system, it is possible to approximate $n_{0} \approx$ in the fluctuation corrections $n_{g}^{(0)}(n)$, $n_{g}^{(T)}(n)$ and $n_{g}^{(\mathrm{dis})}(n)$. Thus, in the following, we are going to approximate $n_{0}$ with $n$ in the perturbativelycomputed fluctuation contributions. In Eq. (31), $n_{g}^{(0)}$ and $n_{g}^{(T)}$ are computed similarly to Eq. (30): first, one has to extract the regularized Gaussian corrections from Eq. Eq. (20), then derive with respect to the chemical potential.

In [33], an extensive analysis addressed this topic for the pure system. Being interested in the role played by the disorder, we report here only the final result, i.e.

$$
\begin{aligned}
n_{g}^{(0)}(n) & =\frac{1}{4 \pi^{d / 2} \Gamma(d / 2)}\left(\frac{m}{\hbar^{2}}\right)^{d / 2} \frac{\left(g_{0} n\right)^{d / 2}}{\lambda^{(d-1) / 2}\left(n, g_{2}\right)}\left[2 \frac{1+\frac{2 m}{\hbar^{2}} g_{2} n}{\lambda\left(n, g_{2}\right)} \mathcal{B}\left(\frac{d+1}{2},-\frac{d}{2}\right)+\mathcal{B}\left(\frac{d-1}{2}, \frac{2-d}{2}\right)\right] \\
n_{g}^{(T)}(n) & =\int \frac{d^{d} \mathbf{q}}{(2 \pi)^{d}} \frac{\frac{\hbar^{2} q^{2}}{2 m}\left(1+\frac{2 m}{\hbar^{2}} g_{2} n\right)+g_{0} n}{\sqrt{\frac{\hbar^{2} q^{2}}{2 m}\left[\lambda\left(n, g_{2}\right) \frac{\hbar^{2} q^{2}}{2 m}+2 g_{0} n\right]}}\left(\frac{1}{e^{\beta E_{q}(n)}-1}\right)
\end{aligned}
$$

with $\mathcal{B}(x, y)$ the Euler beta function, which can be rewritten, after analytic continuation, in terms of the Euler gamma function $\Gamma(x)$ as follows $\mathcal{B}(x, y)=$ $\Gamma(x) \Gamma(y) / \Gamma(x+y) . \quad E_{q}(n)$ is the spectrum of collective excitations given by Eq. (19) with $n_{0} \approx n$.

\section{ANALYTICAL AND NUMERICAL RESULTS IN THREE DIMENSIONS}

We now consider the common situation of bosons moving in three spatial dimensions, i.e. $d=3$. In this case, during the calculation of $\Omega(v, \mu)$, only power divergences arise. Within the dimensional regularization scheme, they are set to zero and no explicit renormalization is required [32]; this holds for both the pure and disorder terms.

\section{A. Condensate density}

By taking the limit $d \rightarrow 3$ in Eqs. (30) and (32), at zero temperature one gets

$$
\begin{aligned}
\frac{n_{0}}{n} & =1-\frac{32}{3 \sqrt{\pi}} \frac{\sqrt{n a_{s}^{3}}}{1+8 \pi\left(n a_{s}^{3}\right)\left(\frac{r_{s}}{a_{s}}\right)}\left[\frac{1+4 \pi\left(n a_{s}^{3}\right)\left(\frac{r_{s}}{a_{s}}\right)}{1+8 \pi\left(n a_{s}^{3}\right)\left(\frac{r_{s}}{a_{s}}\right)}-\frac{3}{4}\right] \\
& -\frac{1}{(4 \pi)^{3 / 2}} \frac{\Delta\left(\frac{m^{2}}{\hbar^{4} n^{1 / 3}}\right)}{\left[1+8 \pi\left(n a_{s}^{3}\right)\left(\frac{r_{s}}{a_{s}}\right)\right]^{3 / 2}\left(n a_{s}^{3}\right)^{1 / 6}} .
\end{aligned}
$$

In Fig. 1 we report the behaviour of the condensate fraction $n_{0} / n$ at $T=0$ as a function of the gas parameter $n a_{s}^{3}$ for different values of the effective range and 

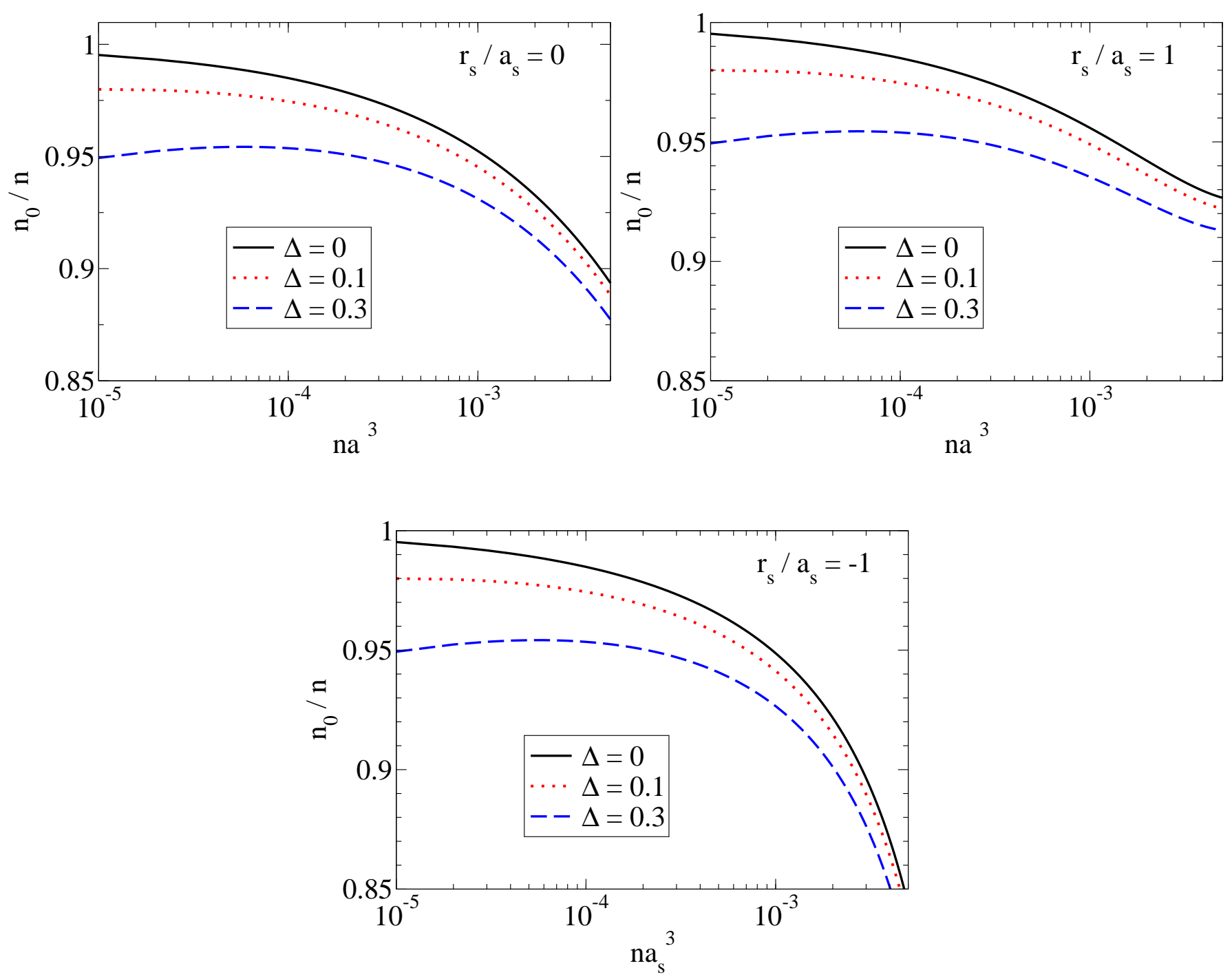

FIG. 1: Condensate fraction $n_{0} / n$ at zero temperature as a function of the dimensionless gas parameter $n a_{s}^{3}$ for increasing disorder strength $\Delta$. The three panels correspond to different values of the ratio $r_{s} / a_{s}$ between the effective range $r_{s}$ and the scattering length $a_{s}$. In the legend, $\Delta$ is expressed in units of $E_{B}^{2} / n$ with $E_{B}=\hbar^{2} n^{2 / 3} / m$. The curves are obtained from Eqs. (32) and (33), which provide a reliable thermodynamic picture of the system within the range of diluteness (i.e. the values of $n a_{s}^{3}$ ) chosen for the horizontal axis.

increasing the disorder correlator $\Delta$. We immediately realize that the weight of the finite-range corrections is enhanced at higher densities (for a given s-wave scattering length). Indeed, from the middle and the right panel of Fig. 1 one can observe that, depending on the sign of the ratio $r_{s} / a_{s}$, the condensate is depleted slower (for $r_{s} / a_{s}>0$ ) or faster (when the ratio is negative). Moreover, the disorder contribution, depending on $r_{s} / a_{s}$, is relevant also at very low values of $n a_{s}^{3}$, where the particles seem to be much more sensitive to the presence of a disordered external potential. As clearly shown by the dotted red curve and the dashed-dotted blue curve in the panels of Fig. 1, the joint presence of disorder and finiterange corrections modifies the condensate depletion of the system.
Despite the corrections reported in Fig. 1 are tiny in magnitude, calculations in the pure case 10 12 show that the finite-range (Gaussian) contribution to $\Omega(\mu, v)$ removes an artificial thermodynamic instability and consequently expand the applicability range of a Gaussian theory. More precisely, by using Eq. (111) with $\tilde{V}(0) \rightarrow g_{0}$ (i.e. $r_{s} \rightarrow 0$ ), one can express the thermodynamic potential as a function of the chemical potential $\mu$. By recalling that $P(\mu)=-\Omega(\mu) / L^{3}$ and that the uniform configuration is stable for $\partial_{\mu}^{2} P(\mu)>0$, one realizes that the uniform configuration is unstable above a critical value of the chemical potential corresponding to $\left(n a_{s}\right)_{c}^{3} \simeq 0.004$. In 11], analytical results for a Bose gas made of hard spheres (where $r_{s} / a_{s}=2 / 3$ ) are compared to corresponding Path-Integral Ground-State MonteCarlo (MC) Sim- 
ulation [42], finding a very good agreement between theoretical predictions and numerical outcomes.

Moreover, already in [12], a modified version of the GPE including corrections due to Gaussian fluctuations and finite-range interactions was found to reproduce reasonably well numerical simulations based on Diffusion MC methods, rather than its zero-range counterpart. Obviously, refined MC simulation are able to explore the behaviour of (bosonic) quantum gases well beyond the range of validity of the Gaussian (i.e. Bogoliubov) approach, at more dense regime and with higher values of the disorder strengths [43].

\section{B. Superfluid density}

The interplay between disorder and non-local interactions can be effectively understood by analyzing, the depletion of the superfluid density. According to the Landau phenomenological description [45], in presence of a superflow, the total density of the system can be split into

$$
n=n_{s}+n_{n}^{\text {(pure) }}(n)+n_{n}^{(\text {dis })}(n),
$$

with $n_{s}$ the superfluid density and $n_{n}=n_{n}^{\text {(pure) }}+n_{n}^{\text {(dis) }}$ the normal one. The depletion of the superfluid is usually driven by the thermal activation of the collective excitations. For instance, in $d=3$ dissipation can occur via density waves (i.e. phonons). As detailed in [29, 46] the original intuition of Landau and Khalatnikov can be adapted to a field-theory analysis, reading the well-known formula

$$
n_{n}^{\text {(pure) }}(n, T)=\frac{\beta}{4 d} \int \frac{d^{d} \mathbf{q}}{(2 \pi)^{d}}\left(\frac{\hbar^{2} q^{2}}{m}\right) \frac{e^{\beta E_{q}}}{\left(e^{\beta E_{q}}-1\right)^{2}}
$$

with $E_{q}$ given, again, by Eq. (19) with $n_{0} \approx n$.

However, it is known that the presence of disorder adds another source of depletion, also at $T=0$. For static point-like defects, this disorder contribution has a peculiar simple form [27, 46], i.e.

$$
n_{n}^{(\mathrm{dis})}(n)=\frac{4}{d} n_{g}^{(\mathrm{dis})}(n)
$$

where $n_{\text {dis }}(n)$ has been computed in Eq. (30). Thus, if $n$ is known, Eqs. (34)- (36) provide an easy way to extract the superfluid fraction of the system. In the case $d=3$ one finds

$$
\frac{n_{s}}{n}=1-\frac{1}{6 \pi^{3 / 2}} \frac{\Delta\left(\frac{m^{2}}{\hbar^{4} n^{1 / 3}}\right)}{\left[1+8 \pi\left(n a_{s}^{3}\right)\left(\frac{r_{s}}{a_{s}}\right)\right]^{3 / 2}\left(n a_{s}^{3}\right)^{1 / 6}}
$$

In Fig. 2 we report the behaviour of $n_{s} / n$, according to the equation above as a function of the gas parameter $n a_{s}^{3}$. In the left panel of Fig. 2 we include the results in absence of a finite-range interaction, in order to better understand the case of $r_{s} \neq 0$.Indeed, for a positive value of the ratio $r_{s} / a_{s}$ (middle panel), the superfluid fraction does not seem to be significantly affected. On the contrary, for a negative value of the effective range $r_{s}$, the left panel of Fig. 2 shows that the behavior of $n_{s} / n$ is no more monotonous within the range of values we have considered for $n a_{s}^{3}$. This an example of the interplay between between disorder and finite-range interactions, both affecting a relevant transport quantity such as the superfluid fraction of the system.

For the sake of completeness, we notice that from Eq. (37) one can also deduces the critical disorder strength

$$
\Delta_{c}=6 \pi^{3 / 2} \frac{\hbar^{4} n^{1 / 3}}{m^{2}}\left[1+8 \pi\left(n a_{s}^{3}\right)\left(\frac{r_{s}}{a_{s}}\right)\right]^{3 / 2}\left(n a_{s}^{3}\right)^{1 / 6}
$$

above which the superfluidity is destroyed despite the absence of thermal excitations. The formula shows that the effective range $r_{s}$ induces a nonlinear shift on $\Delta_{c}$.

\section{CONCLUSIONS}

We have considered a bosonic system with a finiterange two-body interaction placed in a disordered environment. We have investigated the superfluid phase of this system and, according to our perturbative fieldtheoretical analysis valid in any spatial dimension $d$, we have computed explicitly, Eqs. (33) and (37), the modified depletion of the condensate and superfluid density in the three-dimensional case. Our results show that disorder and non-local interactions simultaneously modify the contribution of quantum and thermal fluctuations, leading to nonuniversal corrections. These theoretical predictions become very important when the s-wave effective range $r_{s}$ of the inter-atomic potential is of the same order (or larger) with respect to the s-wave scattering length $a_{s}$. This regime can be achieve by approaching a zero-point crossing of the scattering where the effective range (otherwise constant and $\sim 10 a_{0}$, with $a_{0}$ the Bohr's radius) may vary and change its sign. It has been pointed out 39] that, in this regime, a single-channel approximation [38] provides quite reliable results.

In lower spatial dimensions quantum and thermal fluctuations are strongly enhanced and a proper characterization of their contribution is mandatory, also in terms of interaction parameters. Moreover, the resulting thermodynamic picture may serve as a starting point to build an effective Gross-Pitaveskii-like equation in the spirit of the local-density-approximation. This has been done, for instance, with strongly magnetic atoms [47, 48], binary mixtures [49] or spin-orbit coupling [50]. However, all these papers does not consider a disordered environment which crucially alters the superfluid dynamics of a condensed systems. The physical picture becomes more richer if we allow the possibility of a localized phase but first we need to understand the interplay between disor- 

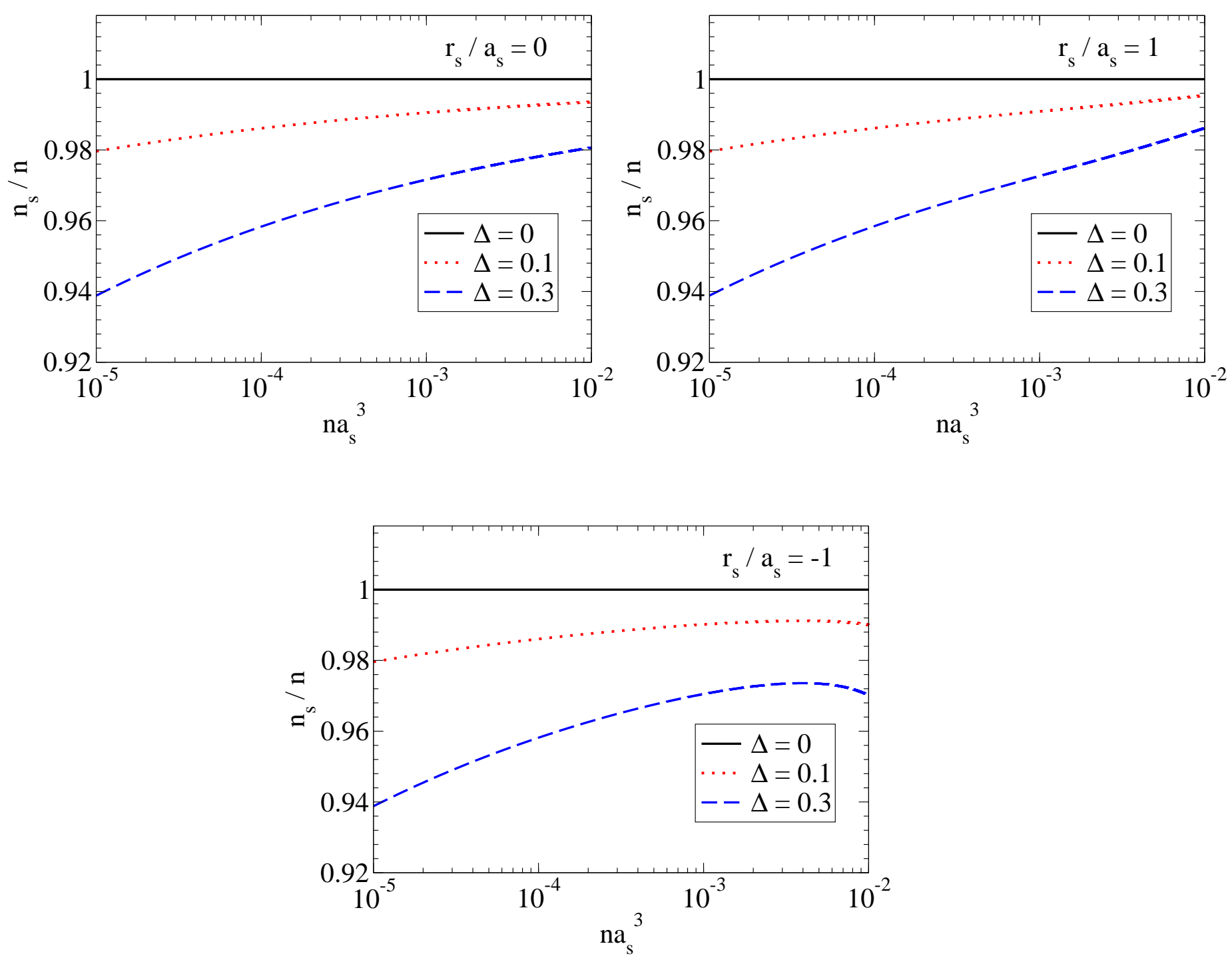

FIG. 2: Superfluid fraction $n_{s} / n$ as a function of the dimensionless gas parameter $n a_{s}^{3}$, for different combinations of $\Delta$ and the ratio $r_{s} / a_{s}$. As in Fig. 1] the disorder strength $\Delta$ is expressed in units of $E_{B}^{2} / n$ with $E_{B}=\hbar^{2} n^{2 / 3} / m$ and $r_{s} / a_{s}$ is the ratio between the effective range $r_{s}$ and the scattering length $a_{s}$. The curves have been obtained from Eq. (37).

der and non-local interaction in the fluctuations contribution, which may help (or not) in driving the system towards the superfluid-to-localized transition.
[1] P. W. Anderson, Absence of diffusion in certain random lattices, Phys. Rev. 109, 1492 (1959).

[2] M. Lewenstein, A. Sanpera, V. Ahufinger, B. Damski, A. Sende, and U. Sen, Ultracold atomic gases in optical lattices: mimicking condensed matter physics and beyond, Advances in Physics 56, 243-379 (2007).

[3] I. Bloch, J. Dalibard, and W. Zwerger, Many-body physics with ultracold gases, Rev. Mod. Phys. 80, 885 (2008).

[4] B. Damski, J. Zakrzewski, L. Santos, P. Zoller, and M. Lewenstein, Atomic Bose and Anderson Glasses in Optical Lattices, Phys. Rev. Lett. 91, 080403 (2003).

[5] T. Schulte, S. Drenkelforth, J. Kruse, W. Ertmer, J. Arlt,
K. Sacha, J. Zakrzewski, and M. Lewenstein, Routes Towards Anderson-Like Localization of Bose-Einstein Condensates in Disordered Optical Lattices, Phys. Rev. Lett. 95, 170411 (2005).

[6] J. C. Dainty (edited by), Laser Speckle and Related Phenomena (Springer, Berlin, 1975).

[7] J. E. Lye, L. Fallani, M. Modugno, D. S. Wiersma, C. Fort and M. Inguscio, Bose-Einstein condensate in a random potential, Phys. Rev. Lett. 95, 070401 (2005).

[8] D. Clement, et al., Suppression of transport of interacting elongated Bose-Einstein condensates in a random potential, Phys. Rev. Lett. 95, 170409 (2005). 
[9] E. Braaten and A. Nieto, Quantum corrections to the energy density of a homogeneous Bose gas, Eur. Phys. J. B 11, 143-159 (1999).

[10] E. Braaten, H.-W. Hammer, and S. Hermans, Nonuniversal effects in the homogeneous Bose gas, Phys. Rev. A 63, 063609 (2001).

[11] A. Cappellaro and L. Salasnich, Thermal field theory of bosonic gases with finite-range effective interaction, Phys. Rev. A 95, 033627 (2017).

[12] H. Fu, Y. Wang, and B. Gao, Beyond the Fermi pseudopotential: A modified Gross-Pitaevskii equation, Phys. Rev. A 67, 053612 (2003).

[13] N. T. Zinner and M. Thogersen, Stability of a BoseEinstein condensate with higher-order interactions near a Feshbach resonance, Phys. Rev. A 80, 023607 (2009).

[14] H. Veksler, S. Fishman and W. Ketterle, Simple model for interactions and corrections to the Gross-Pitaevskii equation, Phys. Rev. A 90, 023620 (2014).

[15] F. Sgarlata, G. Mazzarella, and L. Salasnich, Effectiverange signatures in quasi-1D matter waves: sound velocity and solitons, J. Phys. B: At. Mol. Opt. Phys. 48, 115301 (2015).

[16] V. Heinonen, K. J. Burns, and J. Dunkel, Quantum hydrodynamics for supersolid crystals and quasicrystals, Phys. Rev. A 99, 063621 (2019).

[17] H. T. C. Stoof, D. B. M. Dickerscheid, and K. Gubbels, Ultracold Quantum Fields (Springer, Dordrecht, 2009).

[18] S. Giorgini, L. Pitaevskii, and S. Stringari, Effects of disorder in a dilute Bose gas, Phys. Rev. B 49, 12938 (1994).

[19] G. M. Falco, A. Pelster, and R. Graham, Thermodynamics of a Bose-Einstein condensate with weak disorder, Phys. Rev. A 75, 063619 (2007).

[20] G. M. Falco, A. Pelster, and R. Graham, Collective excitations in trapped Bose-Einstein condensed gases in the presence of weak disorder, Phys. Rev. A 76, 013624 (2007).

[21] S. F. Edwards and P. W. Anderson, Theory of spin glasses, J. Phys. F 5, 965974 (1975).

[22] M. Mezard, G. Parisi, and M. Virasoro, Spin Glass Theory and Beyond: An Introduction to the Replica Method and Its Applications (World Scientific, Singapore, 1987).

[23] G. Parisi, On the replica approach to random directed polymers in two dimensions, J. Phys. France 51, 1595 (1990).

[24] M. Mezard and G. Parisi, Replica Field Theory for Random Manifolds, J. Phys. I France 1, 809 (1991).

[25] R. Graham and A. Pelster, Order via Nonlinearity in Randomly Confined Bose Gases, Int. J. Bif. Chaos 19, 2745 (2009).

[26] D. R. Nelson and P. Le Doussal, Correlations in flux liquids with weak disorder, Phys. Rev. B 42, 10113 (1990).

[27] A. Cappellaro and L. Salasnich, Superfluids, Fluctuations and Disorder, Appl. Sci. 9, 1498 (2019).

[28] A. Altland and B. Simons, Condensed Matter Field Theory (Cambridge University Press, Cambridge, 2010).

[29] U. Taüber and D. R. Nelson, Superfluid bosons and flux liquids: disorder, thermal fluctuations, and finite-size effects, Phys. Rep. 289, 157-233 (1997).
[30] G. M. Falco, T. Nattermann and V. L. Pokrovsky, Weakly interacting Bose gas in a random environment, Phys. Rev. B 80, 104515 (2009).

[31] T. Haugset, H. Haugerud, and F. Ravndal, Thermodynamics of a weakly interacting Bose-Einstein gas, Annals of Physics 266, 27-62 (1997).

[32] J. O. Andersen, Theory of the weakly interacting Bose gas, Rev. Mod. Phys. 76, 599 (2004).

[33] A. Tononi, A. Cappellaro, and L. Salasnich, Condensation and superfluidity of dilute Bose gases with finiterange interaction, New J. Phys. 20, 125007 (2018).

[34] G. M. Bruun, A. D. Jackson and E. E. Kolomeitsev, Multichannel scattering and Feshbach resonances: Effective theory, phenomenology, and many-body effects, Phys. Rev. A 71, 052713 (2005).

[35] S. Kokkelmans, et al., Resonance superfluidity: Renormalization of resonance scattering theory, Phys. Rev. A 65, 053617 (2002).

[36] R. Duine and H. T. C. Stoof, Atom-molecule coherence in Bose gases, Phys. Rep. 396, 115 (2005).

[37] L. Pricoupenko, Many Bosons in a Narrow Magnetic Feshbach Resonance, Phys. Rev. Lett. 110, 180402 (2013).

[38] B. Gao, Analytic description of atomic interaction at ultracold temperatures. II. Scattering around a magnetic Feshbach resonance, Phys. Rev. A 84, 022706 (2011).

[39] C. L. Blackley, P. S. Julienne and J. Hutson, Effectiverange approximations for resonant scattering of cold atoms, Phys. Rev. A 89, 042701 (2014).

[40] A. J. M. Schakel, Quantum critical behavior of disordered superfluids, Phys. Lett. A 224, 287-292 (1997).

[41] E. Zeidler, Quantum Field Theory II. Quantum Electrodynamics (Springer-Verlag, Berlin, 2009).

[42] M. Rossi and L. Salasnich, Path-integral ground state and superfluid hydrodynamics of a bosonic gas of hard spheres Phys. Rev. A 88, 053617 (2013).

[43] S. Pilati, S. Giorgini, M. Modugno and N. Prokof'ev, Dilute Bose gas with correlated disorder: a path integral Monte Carlo study, New J. of Phys. 12, 073003 (2010).

[44] L. Salasnich and F. Toigo, Zero-point energy of ultracold atoms, Phys. Rep. 640, 1 (2016).

[45] L. D. Landau and E. M. Lifshitz, Statistical Physics 2 (Pergamon Press, Oxford, 1987).

[46] A. M. J. Schakel, Boulevard of Broken Symmetries (World Scientific, Singapore, 2008).

[47] F. Wachtler and L. Santos, Quantum filaments in dipolar Bose-Einstein condensates, Phys. Rev. A 93, 061603 (2016).

[48] R. N. Bisset, R. M. Wilson, D. Baillie, and P. B. Blakie, Ground-state phase diagram of a dipolar condensate with quantum fluctuations, Phys. Rev. A 94, 033619 (2016).

[49] G. E. Astrakharchik and B. A. Malomed, Dynamics of one-dimensional quantum droplets, Phys. Rev. A 98, 013631 (2018).

[50] A. Tononi, Y. Wang, and L. Salasnich, Quantum solitons in spin-orbit-coupled Bose-Bose mixtures, Phys. Rev. A 99, 063618 (2019). 